\title{
Anabases
}

ANABASES Traditions et réceptions de l'Antiquité

$21 \mid 2015$

Varia

\section{Les traductions latines du théâtre grec. Introduction}

Malika Bastin-Hammou

\section{(2) OpenEdition}

1 Journals

Édition électronique

URL : http://journals.openedition.org/anabases/5219

DOI : 10.4000/anabases.5219

ISSN : 2256-9421

Éditeur

E.R.A.S.M.E.

Édition imprimée

Date de publication : 1 avril 2015

Pagination : 41-44

ISSN : 1774-4296

\section{Référence électronique}

Malika Bastin-Hammou, « Les traductions latines du théâtre grec. Introduction », Anabases [En ligne],

21 | 2015, mis en ligne le 01 avril 2018, consulté le 21 octobre 2019. URL : http://

journals.openedition.org/anabases/5219; DOI : 10.4000/anabases.5219

(c) Anabases 


\section{Les traductions latines du théâtre grec. Introduction}

Malika Bastin-Hammou

$\mathrm{L}$

es textes réunis ici résultent d'un colloque sur les Traductions savantes vers le latin qui s'est déroulé en novembre 2013 à Lyon. La première journée, plus spécifiquement tournée vers des questions d'histoire du livre et du latin, fera l'objet d'une publication à part; la seconde, dont les textes qui suivent sont issus, portait sur les traductions latines du théâtre grec.

De leur redécouverte $\mathrm{au} \mathrm{Xv}^{\mathrm{e}}$ siècle jusqu'au $\mathrm{xIx}^{\mathrm{e}}$ siècle, les textes dramatiques grecs ont fait l'objet de traductions latines, alors même que, avec le temps, se multipliaient les traductions en vernaculaires. Or si des travaux ont été consacrés à la tradition de tel ou tel poète dramatique ${ }^{1}$, englobant certaines traductions latines de leur œuvre, le corpus des traductions en latin du théâtre grec en tant que tel n'a à ce jour jamais fait l'objet de travaux.

Or ces textes constituent un corpus spécifique qui mérite qu'on s'y intéresse en propre. Tous sont des textes en vers, et posent la question de la transposition métrique: faut-il traduire en vers ou en prose? Et si l'on traduit en vers, quel vers latin choisir pour correspondre à tel vers grec, notamment dans la traduction des chœurs? Faut-il respecter seulement les types de vers de l'original, ou aller jusqu'à en reproduire les pieds? Selon les lieux, les périodes, et les intentions des traducteurs, la réponse varie. Si Leonardo Bruni, qui traduit vers I 440 le prologue du Ploutos d'Aristophane, semble avoir d'abord choisi de traduire en

1 E. Borza, Sophocles Redivivus. La survie de Sophocle en Italie au début duxvie siècle. Éditions grecques, traductions latines et vernaculaires, Bari, Levante editori, 2007; M.MundDöpchie, La survie d'Eschyle à la Renaissance. Édition, traductions, commentaires et imitations, Louvain, Peeters, I984. 
vers puis abandonné pour passer à la prose, ses successeurs font le plus souvent le choix de traduire en vers et s'en glorifient: leurs pages de titre le mentionnent et leurs préfaces soulignent les efforts que cela leur a coûté. Certains cependant font exception - ceux qui sont davantage soucieux de fournir, avec leur traduction, un outil utile à la compréhension du grec qu'un texte agréable à lire et “à la hauteur » de l'original. En font partie les traductions ad verbum - comme celle, pour Aristophane, d'Andrea Divo. Première traduction complète de l'auteur comique (I538), elle est accueillie avec enthousiasme par les lecteurs, comme en témoignent ses nombreuses réimpressions; mais elle est également la cible des sarcasmes des érudits qui lui succèdent ${ }^{2}$. Charles Girard aussi, qui fut professeur de grec à l'université de Bourges, se soucie peu de métrique. Il fait paraître à Paris, en I549, une traduction du Ploutos en prose, agrémentée de nombreux commentaires très utiles à la compréhension du texte: il avait davantage à cœur d'expliquer pas à pas l'original grec que de fournir un texte destiné à une lecture en continu, à preuve la mise en page qu'il a adoptée. Quelques vers grecs sont suivis de leur traduction latine, puis de leur commentaire (voir figure I), là où les traducteurs faisant le choix de traduire en vers soit ne publient que le texte latin - c'est le cas de la traduction du Ploutos de Miguel Cabedio, parue à Paris en 1547 - soit proposent des éditions bilingues avec une rigoureuse correspondance vers à vers du texte latin et du texte grec $^{3}$. Ainsi procèdent Nicodemus Frischlinus ou encore Florent Chrestien dans leurs traductions d'Aristophane, et les éditions du xvII ${ }^{\mathrm{e}}$ siècle, qui reprennent les traductions de Divo, Frischlin et Chrestien sont toutes des bilingues. Mais quand Stephanus Bergler, en I76o, fait paraître une nouvelle édition d'Aristophane, il remplace les traductions de Frischlin et Chrestien, considérées comme trop «littéraires ", par des traductions en prose. Et dans l'édition de Brunck de i78I, le texte est accompagné d'une nouvelle traduction en prose de l'ensemble des comédies, dont il est le seul auteur. La fidélité métrique n'est plus à l'ordre du jour, du moins chez les savants.

De la même manière, les traducteurs vont peu à peu s'émanciper du modèle que constitue pour beaucoup le théâtre latin. Tous abordent en effet le théâtre grec avec en tête leur connaissance du théâtre latin. Mais si certains s'en inspirent, voire s'en réclament, d'autres tentent au contraire de le tenir à distance. Ainsi Érasme, qui est le premier à avoir traduit une tragédie grecque complète, l'Hécube

2 Voir, dans ce volume, la contribution de Simone Beta.

3 Voir par exemple l'avis au lecteur de Thomas Venatorius, auteur d'une traduction latine du Ploutos (153i):

"Traduximus bona fide omnia integre,

Ut pene versus versui respondeat,

Adeoque ut ipsum saepe iambum expresserit

Iambus alter, feceritque pes pedem. » 
d'Euripide, en latin, s'efforce-t-il de ne pas s'inspirer de Sénèque, dont le style lui semble trop ampoulé pour rendre justice à l'élégance d'Euripide. Mais Florent Chrestien revendique, aussi bien dans ses traductions de comédies que de tragédies, l'influence du modèle latin: sur la première page de sa traduction de la Paix (I589) il annonce en effet que son édition est «Cum Latina Graeci Dramatis Interpretatione Latinorum Comicorum stylum imitata, \& eodem genere versuum cum Graecis conscripta.», et sur celle des Sept contre Thèbes d'Eschyle (I585), "Stylo ad veteres tragicos Latinos accedente quam proxime fieri potuit». Brunck, en I78I, ne fait plus référence au modèle latin, que ce soit pour le revendiquer ou pour s'en distancer. La page de titre n'affiche que «Aristophanis Comodice in latinum sermonem conversa». Il n'y a pas d'avis au lecteur, de dédicace ni le moindre paratexte dans lequel Brunck aurait pu exposer ses principes de traduction, et l'utilisation du latin semble ici un choix purement linguistique, délié de toute considération stylistique ou générique. C'est, bel et bien, une traduction savante, au sens où elle ne s'adresse plus qu'aux savants - tandis que se multiplient, en cette fin de $\mathrm{xvIII}^{\mathrm{e}}$ siècle, les traductions en vernaculaires de l'auteur comique: la première traduction complète d'Aristophane en français, due à Poinsinet de Sivry, paraît treize ans plus tard, en i784.

De ce point de vue, il convient cependant de distinguer les traductions des tragiques de celles d'Aristophane. La comédie ne connaît pas le même sort que la tragédie, dont les textes sont bien plus rapidement traduits en vernaculaires. Certes, les frères Rositini font paraître dès 1545 une traduction complète d'Aristophane en italien. Mais en France, il faut attendre la fin du xvir ${ }^{\mathrm{e}}$ siècle pour que des comédies d'Aristophane - le Ploutos et les Nuées - soient pour la première fois traduites en français, par les soins de Madame Dacier (I684). En revanche, en ce qui concerne la tragédie, l'Électre de Sophocle est traduite dès i537 par Lazare de Baïf, l'Hécube d'Euripide en i544 par Bochetel et Iphigénie à Aulis en i549 par Sébillet, comme si l'intérêt que représentait pour les dramaturges et gens de théâtre l'accès aux tragédies grecques avait entraîné ces traductions précoces en vernaculaires. C'est d'autant plus probable que ces traductions se concentrent, pendant deux siècles, sur quelques tragédies, avec en tête Edipe-roi, dont la fortune est sans doute à mettre en relation avec l'intérêt porté à la Poétique d'Aristote. Les éditions complètes des poètes tragiques en français, en revanche, sont presqu'aussi tardives que celle d'Aristophane: Sophocle complet paraît en ı762, Eschyle complet en I770 et Euripide complet en 1782 .

Pendant ce temps, ce sont donc des traductions complètes en latin qui circulent, et qui continuent de circuler après la parution des vernaculaires complètes. Les deux visent en effet des publics différents. Tandis que les traductions latines vont peu à peu ne plus s'adresser qu'au public savant, les premières traductions en vernaculaires ont pour ambition de toucher un large public, et notamment celui des gens de théâtre - c'est du moins ce qu'affirme Madame Dacier dans sa Préface à 
son Aristophane français de i684. L'horizon de ces traductions ne semble pourtant pas être celui de la représentation, du moins pas avant le milieu du XIX ${ }^{\mathrm{e}} \mathrm{s}$. Auparavant, s'il arrive que des pièces grecques soient jouées, en grec, en latin ou même en vernaculaires, c'est dans les Collèges. Mais c'est là un autre chapitre de l'histoire de la réception du théâtre grec.

\author{
Malika Bastin-Hammou \\ Maître de conférences en langue \\ et littérature grecques \\ Univ. Grenoble Alpes, LITT\&ARTS \\ F-38040 Grenoble \\ malika.bastin@u-grenoble3.fr
}

ARISTOPHANIS PIVTVS.

KAPI $\Omega$ N.

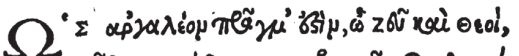

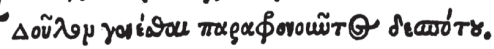

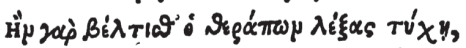

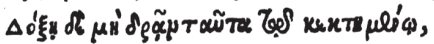

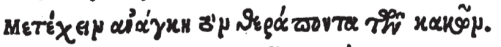

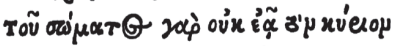

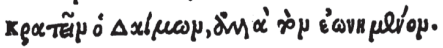

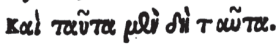

\title{
CARION.
}

Dii boni quàm graue eft infano feruire domino, \& defipienti ! Famulo etenim fi continget vt etiam optima quxq; loquacur, dominus autem ea fecus quàm diCta fuerint accipiat, feruum ineptiarum domini cöfortem fieri omnino neceffe eft. Quandoquidem nefcio qua Fortunx inuidia comparatum videmus, vt is ius obtineat in corpus, non qui corporis eft dominus, fed qui corporis emptor eft. Ethęc, vt dixi, ita mediusfidius fe habent.

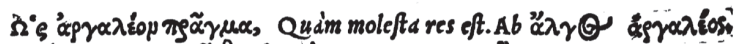

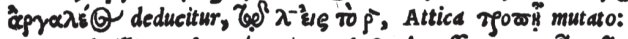

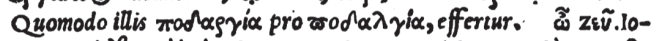

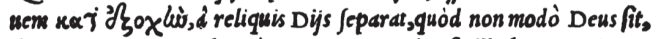
fed Deorum omnium longè maximus. Tale eft illud Homcricum,

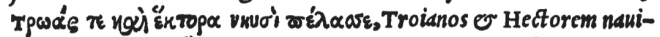

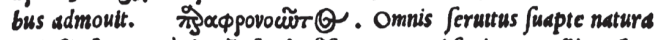
odiofa eft, ea ueró demü eft odiofißßima, qua qui fapit compellitur fer-

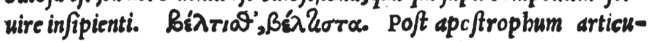
lus \& apiratus precedentem tenuem $T_{2}$ in appiratam $\theta$, mutauit.

$\lambda \xi \xi \alpha \varrho \tau v^{\prime} \chi$. Ad uerbum fonant, dicens fuerit: periphrafis est pro $\lambda \dot{k}^{3}$, dixerit. Est Túx indefinitum $B$, Jubiunctiui , quod dat turyadyw uerbum anorme. Eiufdem modi $\sigma$ temporis eft dó- Túx.

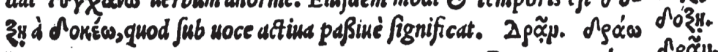

$$
\text { B }
$$

